\title{
Editorial
}

\section{Fixed-Point Theory, Variational Inequalities, and Its Approximation Algorithms}

\author{
Giuseppe Marino, ${ }^{1}$ Vittorio Colao, ${ }^{1}$ Yonghong Yao, ${ }^{2}$ \\ Genaro López ${ }^{3}$ and Enrique Llorens-Fuster ${ }^{3}$ \\ ${ }^{1}$ Dipartimento di Matematica, Universitá della Calabria, 87036 Arcavacata di Rende (CS), Italy \\ ${ }^{2}$ Department of Mathematics, Tianjin Polytechnic University, Tianjin 300160, China \\ ${ }^{3}$ Departemento de Análisis Matemático, Universidad de Sevilla, 41004 Seville, Spain
}

Correspondence should be addressed to Giuseppe Marino, gmarino@unical.it

Received 26 September 2011; Accepted 26 September 2011

Copyright (C) 2011 Giuseppe Marino et al. This is an open access article distributed under the Creative Commons Attribution License, which permits unrestricted use, distribution, and reproduction in any medium, provided the original work is properly cited.

The study of variational inequalities, fixed points and approximation algorithms constituted a topic of intensive research efforts, especially within the past 30 years. As of today, this remains one of the most active fields in mathematics, and its ground of application varies from game theory, economics, engineering, and natural sciences, among others. On the other hand, the nature of many practical problems suggests an iterative approach to the solution.

The aim of this special issue had been to present newest and extended coverage of the fundamental ideas, concepts, and important results on at least one the following topics: iterative schemes to approximate fixed points of nonexpansive-type mappings, iterative approximations of zeros of accretive-type operators, iterative approximations of solutions of variational inequalities problems, iterative approximations of solutions of equilibrium problems, and iterative approximations of common fixed points (and/or common zeros) of families of these mappings.

In the first and very interesting paper by A. Moudafi and E. Al-Shemas, a forward backward algorithm with penalization parameters is introduced and studied for solving variational inequalities in Hilbert spaces. K. Wattanawitoon and P. Kumam studied the weak and strong convergence of iterative methods to a solution of a variational inequality involving an $\alpha$-inverse strongly monotone operator, which is also a zero of a maximal monotone operator and the mixed-equilibrium point of a bifunction $\Theta$. New hybrid algorithms had been analyzed in the papers by J. F. Tan and S. S. Chang, by Y. Shehu, and by J. Zhu and S. S. Chang for solutions of generalized equilibrium problems and fixed points of nonexpansivetype mappings. As for semigroups of nonexpansive mappings, P. Sunthrayuth and P. Kumam introduced a composite iterative scheme for finding a common fixed point in the framework of Banach spaces which admit a weakly continuous duality mapping, while in the paper by 
R. Wangkeeree, a weakened viscosity algorithm is studied for these semigroups. For general families of mappings, iterative procedures had been examinated by S. Chang et al. for families of Lipschitz pseudocontractions, by P. Katchang et al., for strict pseudocontractions, and by Z. Zuo for families of multivalued nonexpansive mappings. Viscosity methods are also studied in the papers by T. Jitpeera and P. Kumam, here used to approximate the unique solution of a minimum problem, and by P. Tianchai for finding solutions of systems of quasivariational inclusions.

J. Chen et al. introduced the concept of Bregman relatively nonexpansive mappings and proved convergence results for the most common iterative schemes. H. Fetter and B. G. de Buen made a comparative study of certain geometric properties of a generic Banach space with basis, endowed with its standard norm as well as with an equivalent norm, similar to the one studied by Lin. Several papers introduce new results about fixed points, variational inequalities, and solutions of equations. Such results may surely lead to new approaches by means of iterative approximations. In particular and for fixed-point theory, I. Altun and A. Erduran presented a fixed-point theorem for a single-valued map in a complete metric space using implicit relations. A. B. Amar et al. proved versions of a fixed-point theorem in a Frechet topological vector space. H. Aydi gave some coupled fixed-point results for mappings satisfying different contractive conditions on complete partial metric spaces. The paper of $\mathrm{M}$. Pemy concerns the optimal selling rule problem and its connection with some variational inequalities. N. B. Yengibaryan and A. G. Barseghyan dealt with solvability and properties of solutions of some homogeneous and nonhomogeneous systems of integral equations. Finally, in the paper by P. Tkacz and M. Turzanski are examined Bolzano-Poincarè type theorems.

\section{Acknowledgments}

The editors would like to thank the authors for their interesting contributions, as well as the Staff and the Editorial Office of the journal for their valuable support. 


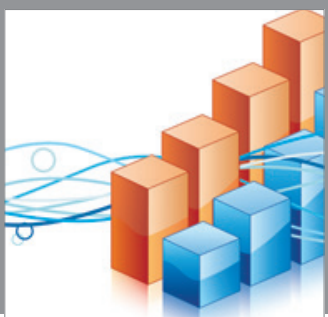

Advances in

Operations Research

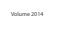

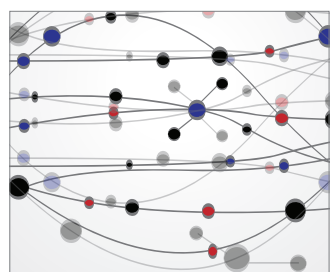

\section{The Scientific} World Journal
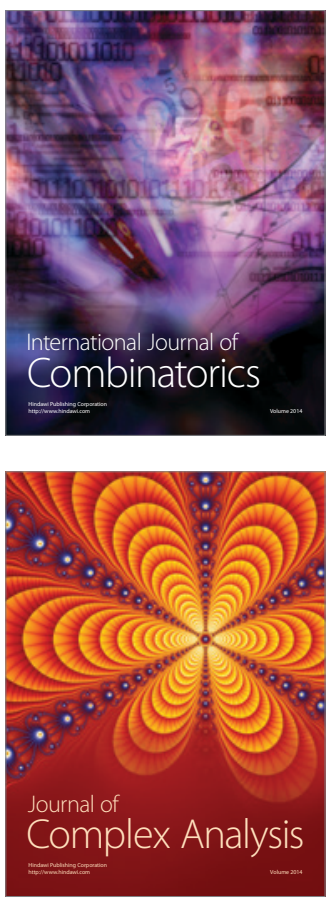

International Journal of

Mathematics and

Mathematical

Sciences
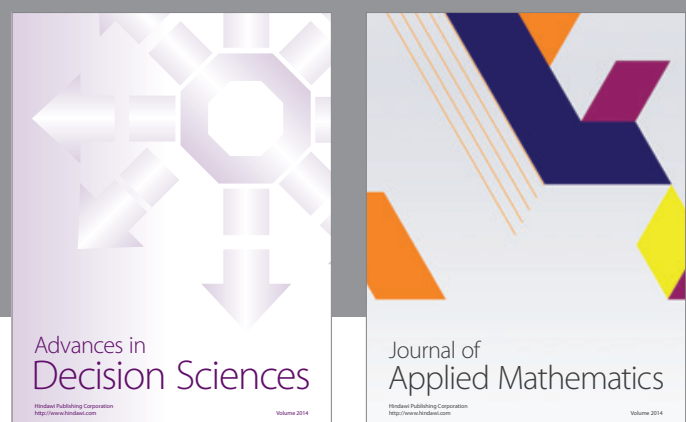

Journal of

Applied Mathematics
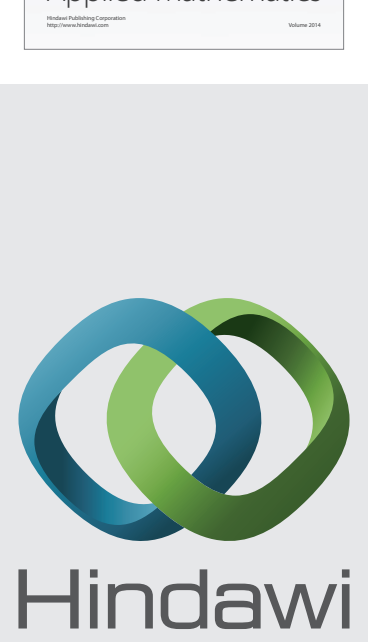

Submit your manuscripts at http://www.hindawi.com
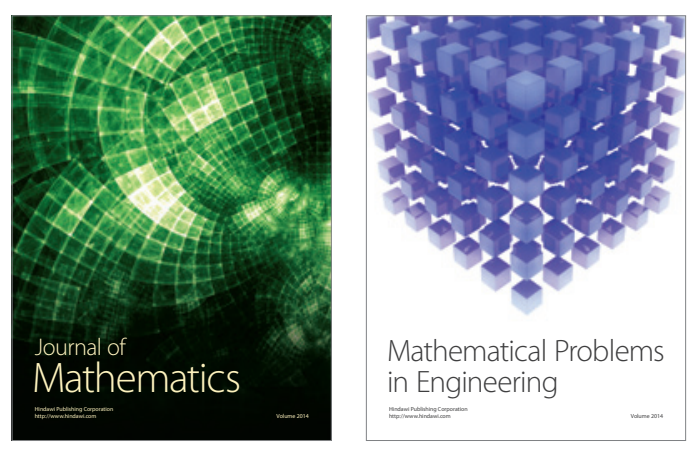

Mathematical Problems in Engineering
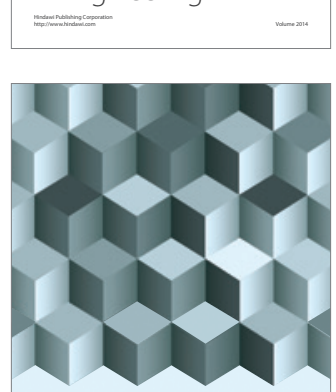

Journal of

Function Spaces
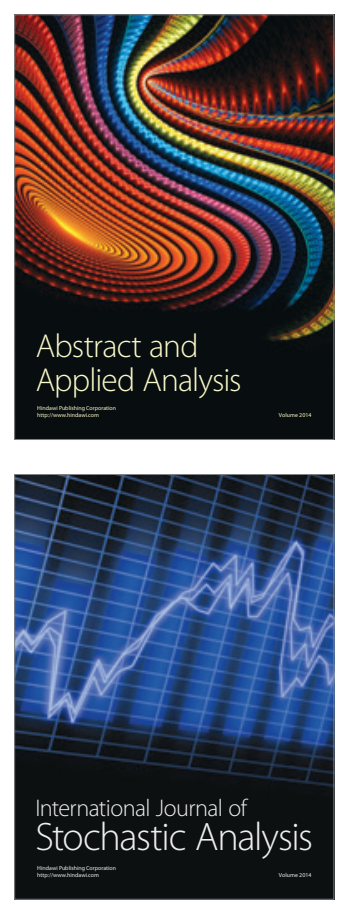

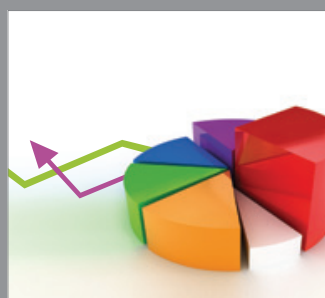

ournal of

Probability and Statistics

Promensencen
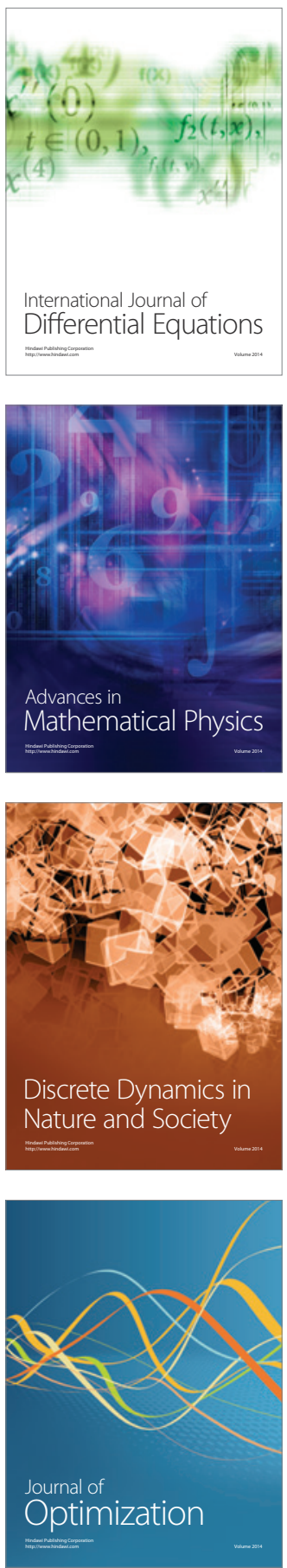